Molecules 2004, 9, 365-372

molecules

ISSN 1420-3049

http://www.mdpi.org

\title{
Nucleophilic Benzoylation Using a Mandelic Acid Dioxolanone as a Synthetic Equivalent of the Benzoyl Carbanion. Oxidative Decarboxylation of $\alpha$-Hydroxyacids
}

\author{
Gonzalo Blay, Isabel Fernández, Belén Monje and José R. Pedro* \\ Departament de Química Orgànica, Facultat de Química, Universitat de València, E-46100 \\ Burjassot(València), Spain. Tel. +34-963544329, Fax +34-963544328.
}

* Author to whom correspondence should be addressed; e-mail: Jose.R.Pedro@uv.es

Received: 5 March 2004 / Accepted: 18 March 2004 / Published: 30 April 2004

\begin{abstract}
The synthesis of alkyl aryl ketones using a mandelic acid dioxolanone as a synthetic equivalent (Umpolung) of the benzoyl carbanion is reported. The methodology involves alkylation of the mandelic acid dioxolanone, hydrolysis of the dioxolanone moiety in the alkylated products and oxidative decarboxylation of the resulting $\alpha$-hydroxyacids. The last step is carried out in a catalytic aerobic way using a Co (III) complex in the presence of pivalaldehyde under very mild conditions.
\end{abstract}

Keywords: Alkylation, dioxolanone, decarboxylation, catalysts, cobalt, Umpolung.

\section{Introduction}

We have recently reported the use of methyl mandelate as a masked $\mathrm{d}^{1}$-synthon for nucleophilic benzoylation ("Umpolung") of the carbonyl group [1] in a synthesis of aryl alkyl ketones, which involves alkylation of methyl mandelate, hydrolysis of the ester group in the alkylated products and oxidative decarboxylation of the resulting $\alpha$-hydroxyacids (Scheme 1). Because of the importance of the carbonyl group in organic chemistry, it is not surprising that several "Umpolung" methods for this group have been described, including the use of dithianes and dithiolanes [2], TosMIC [3], enol ethers [4], vinyl sulfides [5], $\alpha$-aminonitriles [6], cyanohydrins [7], SAMP-hydrazones [8], or heterocycles 
[9] among other. In this paper, we wish to report a related procedure which uses a different mandelic acid derivative, namely 2,2-dimethyl-5-phenyl-1,3-dioxolan-4-one (3) as a new umpoled synthon of the benzoyl carbanion leading to alkyl aryl ketones.

\section{Results and Discussion}

In our earlier work [1] we carried out the alkylation of the dianion of methyl mandelate (2) using 2 equiv. of LDA and 1 equiv. of an alkyl halide as it had been described in the literature [10]. Now, we have carried out the alkylation of a mandelic acid dioxolanone, namely 2,2-dimethyl-5-phenyl-1,3dioxolan-4-one (3), which has the advantage of requiring only one equivalent of strong base (Scheme 1).

Dioxolanone 3 was readily prepared by acid catalysed condensation of mandelic acid (1) with acetone. The lithium enolate derived from $\mathbf{3}$ was alkylated with several organic halides (Table 1). The use of alkyl, allyl or benzyl chlorides as alkylating reagents was unsuccessful as the starting materials were recovered unreacted in all of the cases. Nevertheless the introduction of benzylic or allylic groups could be successfully achieved using the corresponding bromides as alkylating reagents [11], while the introduction of an alkyl group required the use of the corresponding iodide and the addition of $20 \%$ HMPA as cosolvent. Following this methodology we were able to prepare in good yields several $\alpha$ alkylated dioxolanones $\mathbf{5}$ bearing primary, allyl or benzyl groups, and other additional functional groups such as a bromide or a carboxyester.

\section{Scheme 1}

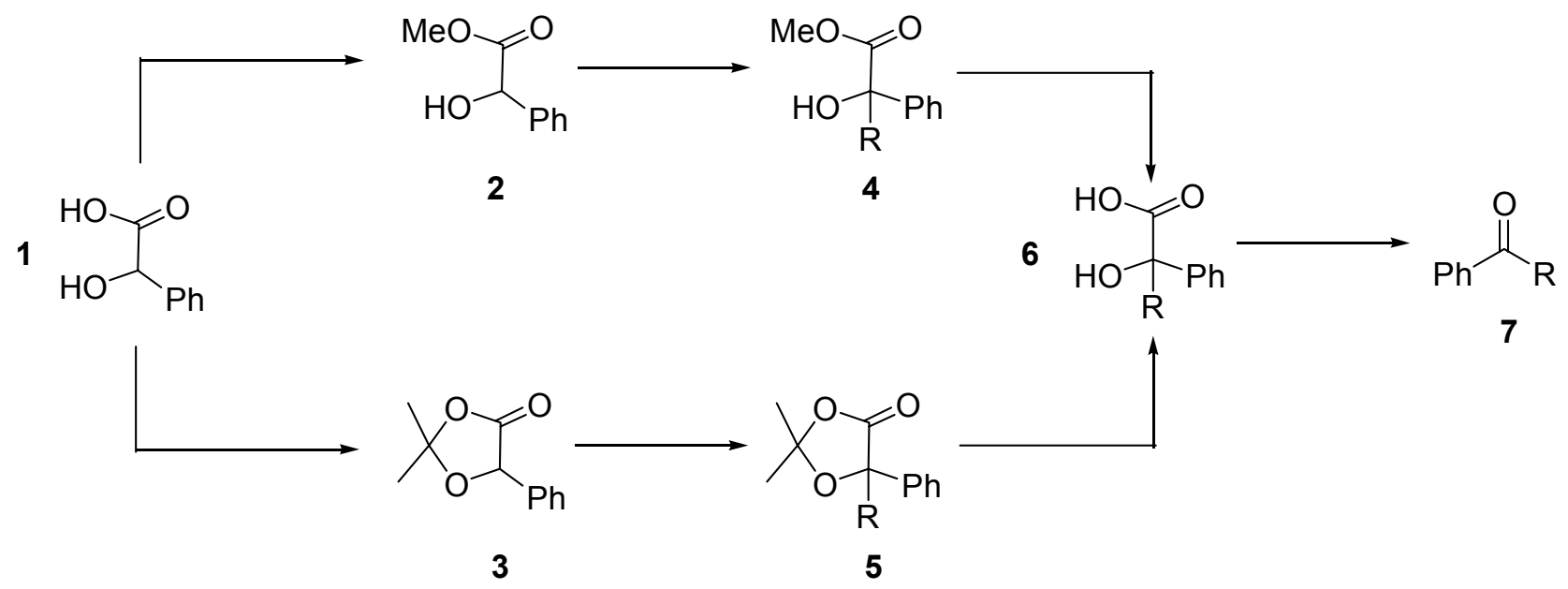


Table 1. Alkylation of 2,2-dimethyl-5-phenyl-1,3-dioxolan-4-one (3) and oxidative decarboxylation of $\alpha$-alkylated mandelic acids (6).

\begin{tabular}{|c|c|c|c|c|c|c|}
\hline Entry & Compound $5^{a}$ & $\begin{array}{r}\text { Yield of } \\
5(\%)\end{array}$ & Compound $6^{b}$ & $\begin{array}{l}\text { Hydrolysis } \\
\text { time (h) }\end{array}$ & Ketone 7 & $\begin{array}{l}\text { Yield of } \\
7(\%)^{c}\end{array}$ \\
\hline
\end{tabular}

a<smiles>CC1(C)OC(=O)C(C)(c2ccccc2)O1</smiles>

b<smiles>CCCC1C(=O)OC2(P)OC(C)(C)OC12P</smiles>

c<smiles>CC(C)=CCCC(C)CCC1(c2ccccc2)OC(C)(C)OC1=O</smiles>

d<smiles>CC1(C)OC(=O)C(Cc2ccccc2)(c2ccccc2)O1</smiles>

e<smiles>CC1(C)OC(=O)C(Cc2ccc(Br)cc2)(c2ccccc2)O1</smiles><smiles>C=CCC1(c2ccccc2)OC(C)(C)OC1=O</smiles><smiles>C=C(Br)CC1(c2ccccc2)OC(C)(C)OC1=O</smiles>

h<smiles>COC(=O)CC1(c2ccccc2)OC(C)(C)OC1=O</smiles>

76<smiles>CC(O)(C(=O)O)c1ccccc1</smiles>

65<smiles>CCCCC(O)(C(=O)O)c1ccccc1</smiles>

62<smiles>CC(C)=CCCC(C)CCC(O)(C(=O)O)c1ccccc1</smiles>

65<smiles>O=C(O)C(O)(Cc1ccccc1)c1ccccc1</smiles>

75<smiles>O=C(O)C(O)(Cc1ccc(Br)cc1)c1ccccc1</smiles>

52<smiles>C=CCC(O)(C(=O)O)c1ccccc1</smiles>
55<smiles>C=C(Br)CC(O)(C(=O)O)c1ccccc1</smiles>

77<smiles>O=C(O)CC(O)(C(=O)O)c1ccccc1</smiles>

6.0<smiles>CC(=O)c1ccccc1</smiles>
87

2.0<smiles></smiles>

2.5<smiles>CC(C)=CCCC(C)CCC(=O)c1ccccc1</smiles>
75

3.0<smiles>O=C(Cc1ccccc1)c1ccccc1</smiles>
93

3.0<smiles>O=C(Cc1ccc(Br)cc1)c1ccccc1</smiles>
94

7.0<smiles>C=CCC(=O)c1ccccc1</smiles>

5.5<smiles>C=C(Br)CC(=O)c1ccccc1</smiles>

9.5<smiles>O=C(O)CC(=O)c1ccccc1</smiles>

${ }^{a}$ Entries a-c required RI as alkylating reagent and the use of 20\% of HMPA as cosolvent. Entries d-h were carried out with $\mathrm{RBr}$ as alkylating reagent.

${ }^{\mathrm{b}}$ Nearly quantitative yields of the hydrolysis products 6 were obtained in all cases.

${ }^{\mathrm{c}}$ The reaction was carried out at room temperature; entries f-g at $0{ }^{\circ} \mathrm{C}$ and entry $\mathrm{h}$ at $-47^{\circ} \mathrm{C}$. 
Upon basic hydrolysis, the products 5 were transformed into the corresponding $\alpha$-hydroxyacids $\mathbf{6}$ in almost quantitative yields. Finally, oxidative decarboxylation of $\mathbf{6}$ gave the corresponding alkyl aryl ketones 7. This transformation was achieved using a catalytic procedure developed in our laboratory which employs molecular oxygen as terminal oxidant in the presence of pivalaldehyde as co-reductant and a catalytic amount of the $\mathrm{Co}(\mathrm{III})$ ortho-phenylene-bis(N'-methyloxamidate) complex [Co(III)$\mathrm{Me}_{2} \mathrm{Opba}$ (Figure 1) [12].

Figure 1

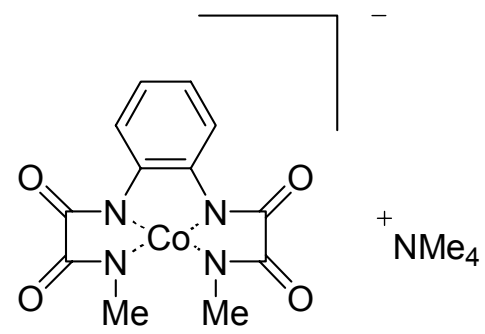

\section{Conclusions}

In summary, we have presented in this paper a procedure for the use of a mandelic acid derivative, namely 2,2-dimethyl-5-phenyl-1,3-dioxolan-4-one (3) as a new masked $\mathrm{d}^{1}$-synthon for the benzoyl group (Umpolung), in which the key step is the aerobic oxidative decarboxylation with oxygen in the presence of pivalaldehyde as co-reductant and the $\mathrm{Co}(\mathrm{III})-\mathrm{Me}_{2} \mathrm{opba}$ complex as catalysts. This reaction is carried out under very mild conditions and it is compatible with the presence in the molecule of other oxidisable groups.

\section{Acknowledgements}

This work was financially supported by the Spanish Government (MCYT, project BQU 20013017) and in part by Generalitat Valenciana. (AVCYT, Grupos 03/168). BM thanks MCYT for a grant.

\section{Experimental}

\section{General}

All melting points are uncorrected. Column chromatography was performed on silica gel 60 (Merck, 0.040-0.063 mm). Unless specified otherwise NMR experiments were run for $\mathrm{CDCl}_{3}$ solutions at $300 \mathrm{MHz}$ for ${ }^{1} \mathrm{H}$ - and at $75 \mathrm{MHz}$ for ${ }^{13} \mathrm{C}-\mathrm{NMR}$, and referenced to the solvent as internal standard. The carbon type was determined by DEPT experiments. Mass spectra were run by electron impact (70 $\mathrm{eV}$ ) or by chemical ionisation using methane as ionising gas. $\mathrm{Co}(\mathrm{III})-\mathrm{Me}_{2} \mathrm{opba}$ complex was prepared according to the previously reported procedure [12]. 
Preparation of 2,2-dimethyl-5-phenyl-1,3-dioxolan-4-one (3)

Conc. $\mathrm{H}_{2} \mathrm{SO}_{4}(1.5 \mathrm{~mL}, 28.36 \mathrm{mmol})$ was added very slowly to a solution of mandelic acid $(2 \mathrm{~g}$, $13.16 \mathrm{mmol})$ in toluene $(160 \mathrm{~mL})$ and acetone $(40 \mathrm{~mL})$. The resulting solution was stirred at room temperature until the reaction was complete ( 24 hours). The reaction mixture was then poured into water $(80 \mathrm{~mL})$, extracted with ether $(3 \times 50 \mathrm{~mL})$, washed with saturated solution of $\mathrm{NaHCO}_{3}$ and brine, and dried over $\mathrm{MgSO}_{4}$. After removal of the solvent under reduced pressure compound 3 (1.64 g, 65\%) was obtained as an oil: ${ }^{1} \mathrm{H}-\mathrm{NMR} \delta$ 7.45-7.35 $(5 \mathrm{H}, \mathrm{m}), 5.38(1 \mathrm{H}, \mathrm{m}), 1.71(3 \mathrm{H}, \mathrm{s}), 1.66(3 \mathrm{H}, \mathrm{s})$; ${ }^{13}$ C-NMR $\delta 171.7$ (s), 134.7 (s), 129.2 (d), 129.0 (d), 126.7 (d), 111.2 (s), 76.1 (d), 27.5 (q), 26.4 (q); MS (EI) $m / z 192\left(\mathrm{M}^{+}, 9.5\right), 177$ (19.6), 148 (35.9), 107 (100), 90 (25.2), 79 (46.8); HRMS (EI) $\mathrm{m} / \mathrm{z}$ required for $\mathrm{C}_{11} \mathrm{H}_{12} \mathrm{O}_{3}$ 192.0786, found 192.0796 .

\section{General procedure for alkylation of 2,2-dimethyl-5-phenyl-1,3-dioxolan-4-one (3)}

A $1.6 \mathrm{M}$ solution of $n$-BuLi in hexane $(0.78 \mathrm{~mL}, 1.25 \mathrm{mmol})$ was added dropwise to a solution of diisopropylamine $(0.175 \mathrm{~mL}, 1.25 \mathrm{mmol})$ in dry THF $(0.8 \mathrm{~mL})$ at $0{ }^{\circ} \mathrm{C}$ under argon. After $30 \mathrm{~min}$, the solution was cooled to $-78{ }^{\circ} \mathrm{C}$ and a solution of dioxolanone $3(192 \mathrm{mg}, 1 \mathrm{mmol})$ in THF $(0.65 \mathrm{~mL})$ was added. The resulting solution was stirred at $-78{ }^{\circ} \mathrm{C}$ for $30 \mathrm{~min}$ and then the corresponding alkyl halide $(1.25 \mathrm{mmol})$ in THF $(0.39 \mathrm{~mL})$ was added. The reaction mixture was stirred at this temperature for 2 hours and then at room temperature until the reaction was complete ( 2 hours). After this time, the reaction mixture was poured into aq. $\mathrm{NH}_{4} \mathrm{Cl}(30 \mathrm{~mL})$, extracted with ether $(3 \times 30 \mathrm{~mL})$, washed with brine and dried over $\mathrm{MgSO}_{4}$. After filtration and removal of the solvent under reduced pressure, the products 5 were obtained by column chromatography (elution with hexane-diethyl ether).

2,2,5-Trimethyl-5-phenyl-1,3-dioxolan-4-one (5a). An oil; ${ }^{1} \mathrm{H}-\mathrm{NMR} \delta 7.54(2 \mathrm{H}, \mathrm{dd}, \mathrm{J}=8.1,1.2 \mathrm{~Hz})$, 7.4-7-2 (3H, m), $1.67(3 \mathrm{H}, \mathrm{s}), 1.61(3 \mathrm{H}, \mathrm{s}), 1.37(3 \mathrm{H}, \mathrm{s}) ;{ }^{13} \mathrm{C}-\mathrm{NMR} \delta 172.7$ (s), 139.8 (s), 127.4 (d), 127.0 (d), 123.5 (d), 109.1 (s), 79.5 (s), 28.2 (q), 27.4 (q), 26.6 (q); MS (EI) $m / z 206$ (M, 6.7$), 191$ (7.8), 162 (83.5), 121 (36.1), 104 (100), 77 (31.8); HRMS (EI) $\mathrm{m} / z$ required for $\mathrm{C}_{12} \mathrm{H}_{14} \mathrm{O}_{3}$ 206.0943, found 206.0949 .

5-Dodecyl-2,2-dimethyl-5-phenyl-1,3-dioxolan-4-one (5b). An oil; ${ }^{1} \mathrm{H}-\mathrm{NMR} \delta 7.57(2 \mathrm{H}, \mathrm{dd}, \mathrm{J}=8.1$, $1.2 \mathrm{~Hz}), 7.4-7.2(3 \mathrm{H}, \mathrm{m}), 1.89(2 \mathrm{H}, \mathrm{m}), 1.64(3 \mathrm{H}, \mathrm{s}), 1.36(3 \mathrm{H}, \mathrm{s}), 1.20(20 \mathrm{H}, \mathrm{m}), 0.83(3 \mathrm{H}, \mathrm{t}, \mathrm{J}=7.0$ $\mathrm{Hz}) ;{ }^{13} \mathrm{C}-\mathrm{NMR} \delta 173.3$ (s), 140.2 (s), 128.3 (d), 127.8 (d), 124.8 (d), 110.0 (s), 83.6 (s), $41.9(\mathrm{t}), 31.9$ $(\mathrm{t}), 29.59(\mathrm{t}$, two overlapped signals), $29.56(\mathrm{t}), 29.48(\mathrm{t}), 29.31(\mathrm{t}), 29.29(\mathrm{t}$, two overlapped signals), 27.8 (q), 24.0 (t), 22.7 (t), 14.1 (q); MS (EI) $m / z 360$ (M , 24.6), 316 (47.9), 275 (28.4), 191 (61.9), 163 (98.7), 105 (100), 77 (33.5); HRMS (EI) $m / z$ required for $\mathrm{C}_{23} \mathrm{H}_{36} \mathrm{O}_{3} 360.2664$, found 360.2650.

2,2-Dimethyl-5-(3,7-dimethyl-6-octenyl)-5-phenyl-1,3-dioxolan-4-one (5c). An oil; ${ }^{1} \mathrm{H}-\mathrm{NMR} \delta 7.56$ $(2 \mathrm{H}, \mathrm{dd}, \mathrm{J}=8.1,1.2 \mathrm{~Hz}), 7.4 .7 .2(3 \mathrm{H}, \mathrm{m}), 5.00(1 \mathrm{H}, \mathrm{m}), 2.0-1.8(4 \mathrm{H}, \mathrm{m}), 1.64(3 \mathrm{H}, \mathrm{s}), 1.61(3 \mathrm{H}, \mathrm{s})$, 
$1.52(3 \mathrm{H}, \mathrm{s}), 1.36(3 \mathrm{H}, \mathrm{s}), 1.35-1.10(5 \mathrm{H}, \mathrm{m}), 0.79(3 \mathrm{H}, \mathrm{t}, \mathrm{J}=6.4 \mathrm{~Hz}) ;{ }^{13} \mathrm{C}-\mathrm{NMR} \delta 173.3(\mathrm{~s}), 140.2(\mathrm{~s})$, $131.2(\mathrm{~s}), 128.3(\mathrm{~d}), 127.8$ (d), 124.8 (d), 124.7 (d), $110.0(\mathrm{~s}), 83.6(\mathrm{~s}), 39.4(\mathrm{t}), 36.7(\mathrm{t}), 32.1(\mathrm{~d})$, 30.8 (t), 27.8 (q), 25.7 (q), 25.3 (t), 19.4 (q), 19.3 (q), 17.6 (q); MS (CI) $m / z 331\left(\mathrm{M}^{+}+1,0.5\right), 301$ (8.1), 273 (100), 245 (15.9), 227 (32.0), 189 (17.7), 137 (13.1), 107 (8.7); HRMS (CI) $m / z$ required for $\mathrm{C}_{21} \mathrm{H}_{31} \mathrm{O}_{3} 331.2273$, found $331.2263\left(\mathrm{M}^{+}+1\right)$.

5-Benzyl-2,2-dimethyl-5-phenyl-1,3-dioxolan-4-one (5d). M.p. 47-49 ${ }^{\circ} \mathrm{C}$ (from $\mathrm{CH}_{2} \mathrm{Cl}_{2}$ ); ${ }^{1} \mathrm{H}-\mathrm{NMR} \delta$ $7.63(2 \mathrm{H}, \mathrm{dd}, \mathrm{J}=8.1,1.2 \mathrm{~Hz}), 7.35-7.22(3 \mathrm{H}, \mathrm{m}), 7.20-7.10(5 \mathrm{H}, \mathrm{m}), 3.31(1 \mathrm{H}, \mathrm{d}, \mathrm{J}=13.9 \mathrm{~Hz}), 2.98$ $(1 \mathrm{H}, \mathrm{d}, \mathrm{J}=13.9 \mathrm{~Hz}), 1.27(3 \mathrm{H}, \mathrm{s}), 1.01(3 \mathrm{H}, \mathrm{s}) ;{ }^{13} \mathrm{C}-\mathrm{NMR} \delta 172.5$ (s), 140.0 (s), 135.0 (s), 131.0 (d), 128.4 (d), 128.0 (d), 127.1 (d), 124.8 (d), 110.4 (s), 84.3 (s), 47.7 (t), 27.9 (q), 27.0 (q); MS (EI) $\mathrm{m} / \mathrm{z}$ $282\left(\mathrm{M}^{+}, 6.4\right), 191$ (96.8), 178 (16.6), 163 (23.1), 105 (100), 77 (45.9); HRMS (EI) $\mathrm{m} / z$ required for $\mathrm{C}_{18} \mathrm{H}_{18} \mathrm{O}_{3} 282.1256$, found 282.1243.

5-(p-Bromobenzyl)-2,2-dimethyl-5-phenyl-1,3-dioxolan-4-one (5e). M.p. $65-66{ }^{\circ} \mathrm{C}$ (from $\mathrm{CH}_{2} \mathrm{Cl}_{2}$ ); ${ }^{1} \mathrm{H}$ NMR $\delta 7.65(2 \mathrm{H}, \mathrm{dd}, \mathrm{J}=8.1,1.2 \mathrm{~Hz}), 7.40-7.30(5 \mathrm{H}, \mathrm{m}), 7.05(2 \mathrm{H}, \mathrm{d}, \mathrm{J}=8.4 \mathrm{~Hz}), 3.29(1 \mathrm{H}, \mathrm{d}, \mathrm{J}=$ $13.9 \mathrm{~Hz}), 3.02(1 \mathrm{H}, \mathrm{d}, \mathrm{J}=13.9 \mathrm{~Hz}), 1.34(3 \mathrm{H}, \mathrm{s}), 1.19(3 \mathrm{H}, \mathrm{s}) ;{ }^{13} \mathrm{C}-\mathrm{NMR} \delta 172.2(\mathrm{~s}), 139.6(\mathrm{~s}), 133.9$ (s), 132.7 (d), 131.1 (d), 128.5 (d), 128.2 (d), 124.8 (d), 121.3 (s), 110.5 (s), 83.9 (s), 47.1 (t), 27.8 (q), 27.2 (q); MS (EI) $m / z$ 362/360 (M+1, 2.0/1.8), 277/275 (7.0/7.4), 191 (100), 178 (25.9), 163 (46.1), 105 (47.8), 77 (99.0); HRMS (EI) $\mathrm{m} / z$ required for $\mathrm{C}_{18} \mathrm{H}_{17} \mathrm{O}_{3} \mathrm{Br} 362.0341 / 360.0361$, found 362.0382 / 360.0360 .

5-Allyl-2,2-dimethyl-5-phenyl-1,3-dioxolan-4-one (5f). An oil; ${ }^{1} \mathrm{H}-\mathrm{NMR} \delta 7.62(2 \mathrm{H}, \mathrm{dd}, \mathrm{J}=8.1,1.2$ $\mathrm{Hz}), 7.40-7.25(3 \mathrm{H}, \mathrm{m}), 5.72(1 \mathrm{H}, \mathrm{m}), 5.15(1 \mathrm{H}, \mathrm{brd}, \mathrm{J}=9.9 \mathrm{~Hz}), 5.13(1 \mathrm{H}, \mathrm{brd}, \mathrm{J}=17.4 \mathrm{~Hz}), 2.70(2 \mathrm{H}$, m), $1.66(3 \mathrm{H}, \mathrm{s}), 1.40(3 \mathrm{H}, \mathrm{s}) ;{ }^{13} \mathrm{C}-\mathrm{NMR} \delta 172.5$ (s), 139.5 (s), 131.3 (d), 128.4 (d), 128.0 (d), 124.8 (d), 120.3 (t), 110.2 (s), 83.3 (s), 45.9 (t), 27.8 (q), 27.7 (q); MS (CI) m/z $233\left(\mathrm{M}^{+}+1,0.8\right), 215$ (2.3), 203 (5.0), 191 (4.5), 175 (100), 157 (7.9), 147 (12.0), 131(30.6) 129 (26.2), 105 (27.5); HRMS (CI) $m / z$ required for $\mathrm{C}_{14} \mathrm{H}_{17} \mathrm{O}_{3} 233.1178$, found $233.1171\left(\mathrm{M}^{+}+1\right)$.

5-(2-Bromoallyl)-2,2-dimethyl-5-phenyl-1,3-dioxolan-4-one (5g). An oil; ${ }^{1} \mathrm{H}-\mathrm{NMR} \delta 7.58(2 \mathrm{H}, \mathrm{dd}, \mathrm{J}=$ 8.1, $1.2 \mathrm{~Hz}), 7.30-7.24(3 \mathrm{H}, \mathrm{m}), 5.61(1 \mathrm{H}, \mathrm{s}), 5.55(1 \mathrm{H}, \mathrm{s}), 3.14(1 \mathrm{H} \mathrm{d}, \mathrm{J}=15.1 \mathrm{~Hz}), 2.93(1 \mathrm{H}, \mathrm{d}, \mathrm{J}=$ $15.1 \mathrm{~Hz}$ ), 1.67 (3H, s), $1.36(3 \mathrm{H}, \mathrm{s}) ;{ }^{13} \mathrm{C}-\mathrm{NMR} \delta 172.0$ (s), 139.0 (s), 128.5 (d), 128.4 (d), $125.2(\mathrm{~s})$, 124.9 (d), 122.9 (t), 110.9 (s), 83.1 (s), 51.6 (t), 27.9 (q), 27.6 (q); MS (CI) $m / z$ 283/281 ( $\mathrm{M}^{+}-\mathrm{C}_{2} \mathrm{H}_{5}$, 3.5/3.9), 255/253 (65.8/70.1), 237/235 (26.7/27.8), 227/225 (22.8/24.0), 191 (64.3), 173 (75.7), 129 (63.8), 105 (100); HRMS (CI) $m / z$ required for $\mathrm{C}_{12} \mathrm{H}_{10} \mathrm{O}_{3} \mathrm{Br} 2872.9793$ / 280.9813, found 282.9757 / 280.9824 ,

2,2-Dimethyl-5-(methoxycarbonylmethyl)-5-phenyl-1,3-dioxolan-4-one (5h). M.p. 105-107 ${ }^{\circ} \mathrm{C}$ (from $\left.\mathrm{CH}_{2} \mathrm{Cl}_{2}\right) ;{ }^{1} \mathrm{H}-\mathrm{NMR} \delta 7.62(2 \mathrm{H}, \mathrm{dd}, \mathrm{J}=8.1,1.2 \mathrm{~Hz}), 7.39-7.27(3 \mathrm{H}, \mathrm{m}), 3.69(3 \mathrm{H}, \mathrm{s}), 3.10(1 \mathrm{H}, \mathrm{d}, \mathrm{J}=$ $16.8 \mathrm{~Hz}), 2.89(1 \mathrm{H}, \mathrm{d}, \mathrm{J}=16.8 \mathrm{~Hz}), 1.66(3 \mathrm{H}, \mathrm{s}), 1.35(3 \mathrm{H}, \mathrm{s}) ;{ }^{13} \mathrm{C}-\mathrm{NMR} \delta 171.9$ (s), 169.0 (s), 138.9 
(s), 128.6 (d), 128.4 (d), 124.7 (d), 110.8 (s), 80.6 (s), 51.9 (q), 45.5 (t), 27.8 (q), 26.7 (q); MS (EI) $\mathrm{m} / \mathrm{z}$ 264 ( $\mathrm{M}^{+}$, 4.4), 220 (31.8), 205 (8.3), 179 (25.6), 133 (21.5), 105 (100), 77 (49.5); HRMS (EI) m/z required for $\mathrm{C}_{14} \mathrm{H}_{16} \mathrm{O}_{5} 264.0998$, found 264.1005.

\section{General procedure for hydrolysis of $\alpha$-alkylated dioxolanones $\mathbf{5}$}

The $\alpha$-alkylated dioxolanones $5(0.3 \mathrm{mmol})$ were treated with $5 \%$ ethanolic $\mathrm{KOH}(0.75 \mathrm{~mL}, 0.6$ $\mathrm{mmol}$ ) at room temperature until complete reaction of the starting material (as indicated by TLC). The solution was poured into ice and acidified with $1 \mathrm{M} \mathrm{HCl}$ to $\mathrm{pH} \sim 2$. The aqueous mixture was extracted with EtOAc $(3 \times 20 \mathrm{~mL})$, the organic layers were washed with brine until neutral, dried, filtered and concentrated under reduced pressure to give the $\alpha$-alkylated mandelic acids 6 in almost quantitative yield. For characterisation of compounds $6 \mathbf{6}, \mathbf{6 b}, \mathbf{6 d}$ and $\mathbf{6 f - 6 h}$ see ref [1].

2-Hydroxy-5,9-dimethyl-2-phenyl-8-decenoic acid (6c). M.p. 78-80 ${ }^{\circ} \mathrm{C}$ (from ethyl acetate); ${ }^{1} \mathrm{H}-\mathrm{NMR}$ $\delta 7.61(2 \mathrm{H}, \mathrm{d}, \mathrm{J}=7.0 \mathrm{~Hz}), 7.40-7.20(3 \mathrm{H}, \mathrm{m}), 5.06(1 \mathrm{H}, \mathrm{m}), 2.3-1.8(4 \mathrm{H}, \mathrm{m}), 1.67(3 \mathrm{H}, \mathrm{s}), 1.57(3 \mathrm{H}, \mathrm{s})$, 1.45-1.10 (5H, m), $0.87(3 \mathrm{H}, \mathrm{t}, \mathrm{J}=6.2 \mathrm{~Hz}) ;{ }^{13} \mathrm{C}-\mathrm{NMR} \delta 180.6(\mathrm{~s}), 141.0(\mathrm{~s}), 131.1(\mathrm{~s}), 128.3(\mathrm{~d}), 128.0$ (d), $125.5(\mathrm{~d}), 124.7(\mathrm{~d}), 78.4(\mathrm{~s}), 37.1(\mathrm{t}), 36.7(\mathrm{t}), 32.3(\mathrm{~d}), 30.3(\mathrm{t}), 25.7(\mathrm{q}), 25.4(\mathrm{t}), 19.4(\mathrm{q})$, 17.6 (q); MS (EI) m/z 290 (M+ 0.8), 272 (49.4), 245 (22.2), 133 (22.3); 110 (77.5), 105 (100), 69 (64.3); HRMS (EI) $m / z$ required for $\mathrm{C}_{18} \mathrm{H}_{26} \mathrm{O}_{3} 290.1882$, found 290.1872 .

3-(p-Bromophenyl)-2-hydroxy-2-phenylpropanoic acid (6e). M.p. $210-212{ }^{\circ} \mathrm{C}$ (from $\mathrm{CH}_{2} \mathrm{Cl}_{2}$ ); ${ }^{1} \mathrm{H}$ NMR (DMSO-d 6 ) $\delta 7.62(2 \mathrm{H}, \mathrm{d}, \mathrm{J}=7.2 \mathrm{~Hz}), 7.40-7.25(5 \mathrm{H}, \mathrm{m}), 7.16(2 \mathrm{H}, \mathrm{d}, \mathrm{J}=7.2 \mathrm{~Hz}) 3.44(1 \mathrm{H}, \mathrm{d}$, $\mathrm{J}=13.6 \mathrm{~Hz}), 3.19(1 \mathrm{H}, \mathrm{d}, \mathrm{J}=13.6 \mathrm{~Hz}) ;{ }^{13} \mathrm{C}-\mathrm{NMR}\left(\right.$ DMSO-d $\left._{6}\right) \delta 175.0(\mathrm{~s}), 142.4(\mathrm{~s}), 136.2(\mathrm{~s}), 132.7$ (d), 130.1 (d), 127.7 (d), 127.1 (d), 125.6 (d), 119.4 (s), 77.8 (s), 44.2 (t); MS (EI) $\mathrm{m} / z$ 304/302 (M - $^{+}$ $\mathrm{H}_{2} \mathrm{O}$, 4.0/3.8), 178 (9.3), 171 (12.3), 169 (13.0), 149 (16.5), 105 (100), 77 (28.8); HRMS (EI) m/z required for $\mathrm{C}_{15} \mathrm{H}_{11} \mathrm{BrO}_{2} 303.9922$ / 301.9942 , found 303.9919 / 301.9937.

General procedure for catalytic aerobic decarboxylation of $\alpha$-hydroxyacids 6

Co (III)-Me 2 opba complex ( $3.3 \mathrm{mg}, 7.7 \times 10^{-3} \mathrm{mmol}$ ) and pivalaldehyde $(46 \mu \mathrm{L}, 0.39 \mathrm{mmol})$ were added to a stirred solution of alkylated $\alpha$-hydroxyacids $6(0.13 \mathrm{mmol})$ in acetonitrile $(0.5 \mathrm{~mL})$ under a dioxygen atmosphere. The mixture was stirred at the indicated temperature until consumption of the starting $\alpha$-hydroxyacid, as indicated by TLC. The reaction products 7 were purified by flash chromatography. For characterisation of compounds $\mathbf{7 a}, \mathbf{7 b}, \mathbf{7 d}$ and $\mathbf{7 f - 7 \mathbf { h }}$ see ref [1].

3,7-Dimethyl-6-octenyl phenyl ketone (7c). An oil; ${ }^{1} \mathrm{H}-\mathrm{NMR} \delta 7.94(2 \mathrm{H}, \mathrm{dd}, \mathrm{J}=8.1$ and $1.2 \mathrm{~Hz}), 7.53$ $(1 \mathrm{H}, \mathrm{tt}, \mathrm{J}=8.1$ and $1.2 \mathrm{~Hz}) 7.43(2 \mathrm{H}, \mathrm{td}, \mathrm{J}=8.1$ and $1.2 \mathrm{~Hz}), 5.08(1 \mathrm{H}, \mathrm{m}), 2.94(2 \mathrm{H}, \mathrm{m}), 1.98(2 \mathrm{H}, \mathrm{m})$, $1.75(2 \mathrm{H}, \mathrm{m}), 1.66(3 \mathrm{H}, \mathrm{s}), 1.58(3 \mathrm{H}, \mathrm{s}), 1.50(2 \mathrm{H}, \mathrm{m}), 1.35(1 \mathrm{H}, \mathrm{m}), 0.92(3 \mathrm{H}, \mathrm{d}, \mathrm{J}=6.2 \mathrm{~Hz})$;

${ }^{13} \mathrm{C}-\mathrm{NMR} \delta 200.8$ (s), 137.0 (s), 132.8 (d), 131.2 (s), 128.5 (d), 128.0 (d), 124.7 (d), 36.9 (t), 36.3 (t), 
$32.2(\mathrm{~d}), 31.3(\mathrm{t}), 25.7(\mathrm{q}), 25.5(\mathrm{t}), 19.4$ (q), 17.6 (q); MS (EI) $\mathrm{m} / z 244\left(\mathrm{M}^{+}, 58.8\right), 201(6.9), 173$ (18.5), 133 (78.9); 122 (77.5), 120 (71.4); 105 (100), 77 (46.6); HRMS (EI) $m / z$ required for $\mathrm{C}_{17} \mathrm{H}_{24} \mathrm{O}$ 244.1827, found 244.1829 .

p-Bromobenzyl phenyl ketone (7e). M.p. $136-138{ }^{\circ} \mathrm{C}$ (from $\left.\mathrm{CH}_{2} \mathrm{Cl}_{2}\right) ;{ }^{1} \mathrm{H}-\mathrm{NMR} \delta 8.00(2 \mathrm{H}, \mathrm{dd}, \mathrm{J}=8.1$, $1.2 \mathrm{~Hz}), 7.65-7.40(5 \mathrm{H}, \mathrm{m}), 7.15(2 \mathrm{H}, \mathrm{d}, \mathrm{J}=8.4 \mathrm{~Hz}), 4.24(2 \mathrm{H}, \mathrm{s}) ;{ }^{13} \mathrm{C}-\mathrm{NMR} \delta 197.2(\mathrm{~s}), 136.6(\mathrm{~s})$, 135.3 (s), 133.6 (d), 132.0 (d), 131.5 (d), 129.0 (d), 128.8 (d), 121.2 (s), 45.0 (t); MS (EI) $\mathrm{m} / 2$ 276/274 $\left(\mathrm{M}^{+}, 9.3 / 9.8\right), 185 / 183$ (15.8/15.0), 171 (9.7), 165 (5.0), 157 (5.8), 105 (73.8), 90 (22.4), 77 (100); HRMS (EI) $m / z$ required for $\mathrm{C}_{14} \mathrm{H}_{11} \mathrm{OBr} 275.9973$ / 273.9993, found 275.9963 / 273.9982.

\section{References}

1. Blay, G.; Fernández, I.; Formentín, P.; Monje, B.; Pedro, J. R.; Ruiz, R. Tetrahedron 2001, 57, 1075 .

2. (a) Seebach, D.; Corey, E.J. J. Org. Chem. 1975, 40, 231; (b) Seebach, D. Synthesis 1969, 17.

3. (a) van Leusen, D.; van Leusen, A.M. Recl. Trav. Chim. Pays-Bas 1991, 110, 393; (b) Moskal, J.; van Leusen, A.M. Tetrahedron Lett. 1984, 25, 2585; (c) Blay, G.; Schrijvers, R.; Wijnberg, J.B.A.; de Groot, A. J. Org. Chem. 1995, 60, 2188.

4. Hartmann, J.; Stähle, M.; Schlosser, M. Synthesis 1974, 888.

5. (a) Corey, E.J.; Seebach, D. J. Org. Chem. 1966, 31, 4097; (b) Carey, F.A.; Court, A.S. J. Org. Chem. 1972, 37, 939.

6. (a) Fabrice, P.; Enders, D. Tetrahedron Lett. 1999, 40, 531; (b) Enders, D.; Mannes, D.; Raabe, G. Synlett 1992, 837.

7. Schrader, T. Chem. Eur. J. 1997, 3, 1273.

8. Enders, D.; Syrig, R.; Raabe, G.; Fernández, R.; Gasch, C.; Lassaleta, J-M.; Llera, J-M. Synthesis 1996, 48.

9. (a) Barco, A.; Benetti, S.; De Risi, C.; Pollini, G.P.; Spalluto, G.; Zanirato, V. Tetrahedron 1996, 52, 4719; (b) Katritzky, A.R.; Oniciu, D.C.; Ghiviriga, I.; Soti, F. J. Org. Chem. 1998, 63, 2110.

10. Ciochetto, L.J.; Bergbreiter, D.E.; Newcomb, M. J. Org. Chem. 1977, 42, 2948.

11. Aitken, R.A.; Thomas, A.W. Synlett 1998, 102.

12. Blay, G.; Fernández, I.; Formentin, P.; Pedro, J.R.; Roselló, A.L.; Ruiz, R.; Journaux, Y. Tetrahedron Lett.1998, 39, 3327.

Samples Availability: Available from the authors.

(C) 2004 by MDPI (http:www.mdpi.org). Reproduction is permitted for noncommercial purposes. 